\title{
Microplásticos nas águas do estuário Tramandaí - Armazém, Litoral Norte do Rio Grande do Sul, Brasil e relação com a qualidade da água
}

\author{
Microplastics in the waters of Tramandaí - Armazém \\ estuary, North Coast Rio Grande do Sul, Brazil and \\ relationship with water quality
}

Laura Steffen Corrêa $^{1}$ (D), Thayse Freitas Silveira ${ }^{1}$ (D), Cacinele Mariana da Rocha² ${ }^{\text {(D), }}$ Daiana Maffessoni ${ }^{1}$ (D)

${ }^{1}$ Universidade Estadual do Rio Grande do Sul - UERGS, Osório, RS, Brasil. E-mails: laurasteffenc@gmail.com, fsthayse35@gmail.com,daiana-maffessoni@uergs.edu.br

${ }^{2}$ Universidade Federal do Rio Grande do Sul - UFRGS, Imbé, RS, Brasil

\begin{abstract}
Como citar: Corrêa, L. S., Silveira, T. F., Rocha, C. M., \&, Maffessoni, D. (2021). Microplásticos nas águas do estuário Tramandaí - Armazém, Litoral Norte do Rio Grande do Sul, Brasil e relação com a qualidade da água. Revista de Gestão de Água da América Latina, 18, e10. https://doi.org/10.21168/rega.v18e10
\end{abstract}

Resumo: A acumulação de partículas de plástico em ambientes aquáticos é acarretada pela produção e uso em grande escala, somada à destinação incorreta enquanto resíduo. Esses pequenos fragmentos, denominados microplásticos (MP), são assunto emergente e considerados um problema mundial, podendo afetar organismos e as suas funções. 0 Litoral Norte do Rio Grande do Sul não possui registros científicos que revelem MP em águas estuarinas. 0 Sistema Estuarino Tramandaí-Armazém (ETA) recebe grande pressão antrópica, especialmente devido ao aglomerado urbano causado pelo turismo, pelos múltiplos usos de suas águas e por ser parte final receptora de águas de toda a Bacia Hidrográfica do Rio Tramandaí (BHRT). Nesse contexto, o objetivo desse estudo foi avaliar a presença de MP nas águas das lagunas Tramandaí e Armazém, visando classificá-los quanto à sua composição polimérica. Além disso, buscou-se estabelecer uma relação entre a ocorrência de MP e a qualidade da água. Os resultados evidenciam ser uma área que apresenta contaminação de média magnitude por MP, sendo que na Laguna do Armazém foi contabilizado um total de 240 und/100 $\mathrm{m}^{3}$ e na Laguna Tramandaí foram determinados 225 und/100 $\mathrm{m}^{3}$ de MP. Destaca-se a predominância de $\mathrm{PE}, \mathrm{PP}$ e PET/PVC que estão estreitamente vinculados às atividades desenvolvidas na região, como a pesca e a ocupação urbana em si. Contudo, não foi possível estabelecer uma relação direta da presença de MP com os parâmetros de qualidade da água. Embora esteja comprovada a contaminação por MP nessa área estuarina, uma avaliação de mais longo período é interessante e pode, inclusive, indicar o efeito, por exemplo, da ocupação massiva durante o veraneio.

Palavras-chave: MP; Contaminação; PET; PVC; Polietileno.

Abstract: The accumulation of plastic in aquatic environments is caused by large-scale production and use, as well as incorrect destination as waste. These small fragments, called microplastics (MP), are emerging issues and considered a worldwide problem once it can affect theirs functions. The North Coast of Rio Grande do Sul does not have scientific records that reveal MP in estuarine waters. The Tramandaí-Armazém Estuary System (ETA) receives great anthropic pressure especially due to the urban agglomeration caused by tourism, to the use of its waters and because it is the final receiver of waters throughout the Tramandaí River Basin (BHRT). The aim of this study was to evaluate the presence of MP in the waters of Tramandaí and Armazém lagoons to classify by polymeric composition. In addition, it was seeked to establish a relationship between the occurrence of MP and the quality of the water. The results show an area that already has medium contamination by MP, which resulted in 240 und MP/100 m³ in Armazém Lagoon and 225 und MP/100 m in Tramandaí Lagoon. The largest amount was PE, PP and PET / PVC, which are closely linked to activities explored in the region, such as fishing and urban occupation. However, it was not possible to establish a direct relationship between the presence of MP and water quality parameters. Although MP contamination is proven in this area, a longer period assessment is interesting and it may indicates the effect, for example, of massive occupation during the summer season.

Keywords: MP; Contamination; PET; PVC; Polyethylene.

Recebido: Janeiro 26, 2021. Revisado: Junho 04, 2021. Aceito: Agosto 09, 2021. 


\section{INTRODUÇÃO}

Sendo definida como subcategoria de uma classe de materiais orgânicos sintéticos poliméricos (Joint Group of Experts on the Scientific Aspects of Marine Environmental Protection, 2015), a palavra "plástico" possui origem grega (plastikós) e tem como significado aquilo que pode ser moldado (Liddell \& Scott, 1889). A produção e o consumo de plástico no mundo vem aumentando a cada ano, estima-se que no ano de 2025, a produção de plástico deverá atingir mais de 600 milhões de toneladas (Montenegro et al., 2020). A crescente demanda por plásticos levou, inevitavelmente, a problemas no descarte de resíduos. Pedaços pequenos desse material foram encontrados flutuando na superfície do oceano e obtiveram relato científico, pela primeira vez, em 1970 (Carpenter \& Smith Junior, 1972; Montenegro et al., 2020).

Presente nas praias, nas águas superficiais, em toda a coluna de água e nos sedimentos, os fragmentos plásticos permearam até os ambientes marinhos mais remotos (Ivar do Sul et al., 2009) e isso é acarretado pela produção e uso em grande escala. Os pequenos fragmentos, denominados microplásticos (MP), possuem menos do que $5 \mathrm{~mm}$ de tamanho, conforme definição da National Oceanic and Atmospheric Administration (NOAA) (Lippiatt et al., 2013), os quais podem ser classificados em primários (esférulas de produção de plástico virgem) e secundários (resultantes da quebra de material plástico de tamanho maior) (Joint Group of Experts on the Scientific Aspects of Marine Environmental Protection, 2015). A abundância de MP seguirá aumentando, fato justificado pelo aumento da fabricação, pelo baixo índice de reciclagem e pela disposição incorreta dos resíduos (Montenegro et al., 2020). Aliado a este fato, o plástico é extremamente durável, de difícil degradação e de fácil flutuação, características que facilitam sua dispersão pelo ambiente (Magalhães \& Araújo, 2012). Portanto, a criação de novas regulamentações é uma forma de controlar a fabricação, venda e distribuição de plásticos a nível mundial, a fim de minimizar a geração desses resíduos (Jiang, 2018). Embora seja um assunto emergente, o MP é considerado um problema mundial, devido ao aumento da produção de plástico e a má gestão dos resíduos sólidos.

Estudos acerca dessa temática estão sendo desenvolvidos em todo mundo. Os MP são comumente estudados quando associados a amostras de plâncton (Cole et al., 2016), sedimentos (Woodall et al., 2014; Alomar et al., 2016), ingestão de vertebrados e invertebrados (Reisser et al., 2014), e interações com poluentes químicos e orgânicos persistentes (Wardrop et al., 2016). De acordo com a literatura disponível, todos os grupos de organismos marinhos estão em um risco iminente de interagir com os MP (Ivar do Sul et al., 2009) e estes podem afetar negativamente os organismos e seu mecanismo físico-químico (Thompson et al., 2004; Lippiatt et al., 2013). Embora uma quantidade considerável de trabalhos esteja focada em MP no meio marinho, estudos relativamente novos sugerem que esses resíduos se apresentam igualmente presentes em águas superficiais, estuários urbanos e efluentes em todo o mundo (Jiang, 2018; Lozoya et al., 2015). Santos et al. (2020) mostraram a ocorrência de microplásticos nas águas superficiais da Bahia, nos Balneários da Prainha e Canto D’Água, sendo esta uma área amplamente empregada por turistas e população local para prática de atividades de recreação e lazer. Silva \& Sousa (2021) evidenciaram a presença de microplásticos na Lagoa dos Patos, lagoa muito importante no sul do Rio Grande do Sul e a maior da América do Sul. Da mesma forma, Bertoldi et al. (2021) verificaram a contaminação por microplásticos em todas as amostras de água no Lago Guaíba, em Porto Alegre.O Litoral Norte do Rio Grande do Sul não possui registros científicos que envolvam MP em águas estuarinas. Essa região tem sido afetada pelo crescimento populacional, o qual contribui com a interferência antrópica. Nos municípios de Imbé e Tramandaí, região costeira nordeste do RS, está localizado o Sistema Estuarino Tramandaí-Armazém (ETA), onde as lagunas recebem contribuição de águas doces e salgadas, ocasionando a formação de um ambiente de água salobra (Castro \& Rocha, 2016). 0 estuário recebe grande pressão antrópica, especialmente devido ao aglomerado urbano causado pelo turismo, pelos múltiplos usos de suas águas e por ser parte final receptora de águas de toda a Bacia Hidrográfica do Rio Tramandaí (BHRT) que ali deságuam encontrando o oceano, de forma que alguns efeitos se manifestam cumulativamente.

Considerando este contexto, entendemos que seja relevante quantificar a presença de MP no ETA, amostrando pontos diferentemente impactados pela ação antrópica, sob diferentes efeitos de ventos e hidrodinâmica, e sua relação com a qualidade da água naquela região. Assim, os dados obtidos fornecerão um panorama pioneiro sobre a contaminação por MP na região do Litoral Norte do RS, além de uma avaliação da qualidade das águas concomitantemente, contribuindo para melhorias da gestão costeira. 


\section{MATERIAL E MÉTODOS}

O Sistema Estuarino Tramandaí-Armazém, localiza-se a $122 \mathrm{Km}$ de Porto Alegre, capital do estado do Rio Grande do Sul, banhando as cidades litorâneas de Tramandaí e Imbé (Figura 1). Região de destino de muitos turistas durante o veraneio, mas também região atrativa para moradores em vista de sua qualidade de vida, o que faz a população oscilar de, aproximadamente, 74 mil habitantes durante o inverno e podendo chegar a mais de 500 mil habitantes durante o verão, no somatório de ambas cidades. Além da presença de moradias localizadas no seu entorno, há também a precariedade nos serviços de saneamento, como o despejo de esgoto não tratado (Castro \& Mello, 2013). Neste local também existe a problematização da pecuária nas margens dos corpos d'água, da pesca sob diversas artes/finalidades e a extração de areia (Castro \& Rocha, 2016).

A lâmina d'água não é muito profunda no sistema estuarino. A Laguna Tramandaí apresenta profundidades média e máxima de $1,0 \mathrm{~m}$ e 1,4 m, respectivamente. Já a Laguna Armazém apresenta profundidade variando entre $0,5 \mathrm{~m}$ e $1,0 \mathrm{~m}$. As taxas de sedimentação de ambas as lagunas situam-se entre 4,2 $\mathrm{mm}$ e 4,4 mm ao ano (Dillenburg, 1994). A distribuição textural dos sedimentos do fundo da Laguna Tramandaí segue o padrão clássico de sedimentação lacustre, com sedimentos mais grossos (areia fina) dominando as áreas rasas e marginais (profundidades menores que $1 \mathrm{~m}$ ) e mais finos (areia lamosa) prevalecendo nas áreas mais profundas. Por outro lado, a Laguna Armazém é quase que inteiramente dominada por areia fina e muito fina. A ausência quase total de sedimentos finos nessa laguna é resultante de sua pequena profundidade (inferior a $1 \mathrm{~m}$ ) e do aporte pouco significativo de siltes e argilas através do Arroio Camarão (Tabajara, 1994). Estudos realizados por Almeida et al. (1997) indicam que a maré astronômica não apresenta grande efeito na região, apresentando uma amplitude entre 0,1 e 0,3 m. Assim, a entrada de água salgada no estuário está condicionada principalmente aos efeitos da pluviosidade e do vento.

O clima da região, segundo a classificação de Köppen, é do tipo subtropical úmido, com temperatura média em torno de $20^{\circ} \mathrm{C}$. 0 padrão de ventos é determinado pela ação de dois centros de alta pressão: o Anticiclone do Atlântico Sul e o Anticiclone Migratório Polar. 0 vento predominante durante o ano é de direção NE, provocado por influência do Anticiclone do Atlântico Sul.

Considerando estas características, as coletas de MP e de amostras de água foram realizadas em 11 pontos, sendo cinco deles (1-5) na Laguna do Armazém e seis pontos (6-11) na Laguna Tramandaí, conforme Figura 1. Esses pontos foram escolhidos para avaliação das possíveis diferenças dentro de múltiplos cenários que se estabelecem no entorno do ETA considerando a influência antrópica como principal fator.

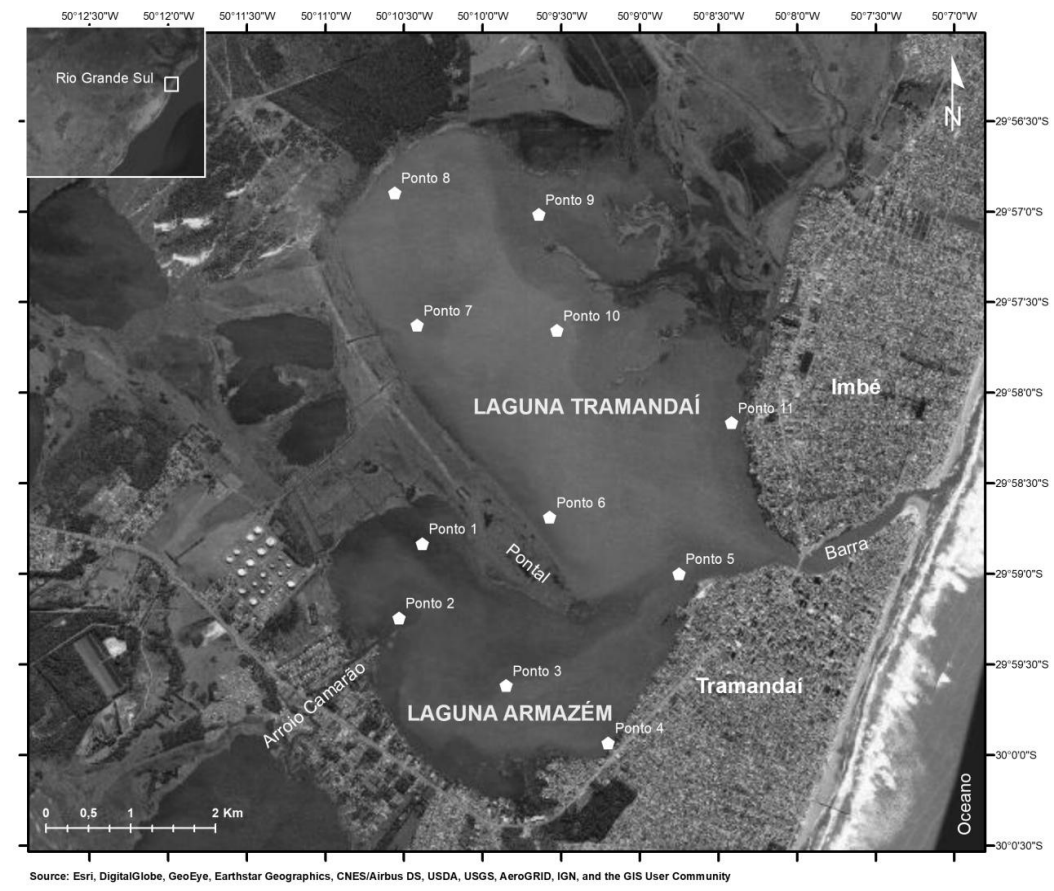

Figura 1. Mapa da área de estudo no Estuário Tramandaí-Armazém, Litoral Norte do RS, identificando os pontos de amostragem.Fonte: Autores (2019). 
As coletas de MP foram realizadas em duas oportunidades, em 16 de janeiro e 22 de julho de 2019, onde foram coletadas respectivamente as amostras das lagunas Armazém e Tramandaí, através de arrastos, em triplicata, utilizando uma rede de plâncton com abertura de malha de $0,3 \mathrm{~mm}$, diâmetro de $0,65 \mathrm{~m}$ e $1 \mathrm{~m}$ de comprimento, método adaptado e recomendado por Lippiatt et al. (2013). Nesse estudo, a hidrodinâmica é avaliada em termos de velocidade da água $(\mathrm{m} / \mathrm{s})$ de água que foi obtido através da utilização de um fluxômetro Oceanic acoplado à rede.

As amostras foram filtradas em malha de $5 \mathrm{~mm}$, armazenadas em béckers e levadas à estufa a $100^{\circ} \mathrm{C}$ para evaporação da água. Posteriormente, foram submetidas à digestão utilizando peróxido de hidrogênio $30 \%$ e catalisador sulfato ferroso $\left(\mathrm{FeSO}_{4}\right)$ em solução, sob aquecimento a $75^{\circ} \mathrm{C}$, a fim de degradar a matéria orgânica presente (Masura et al., 2015). As amostragem realizada foi composta e as quantidades totais de MP a cada $100 \mathrm{~m}^{3}$ foram apresentadas por ponto. Na sequência, os MP foram diferenciados qualitativamente conforme sua classe polimérica em polietileno de alta e baixa densidade (PEAD e PEBD, respectivamente), polipropileno (PP), poliestireno (PS), polietileno de tereftalato (PET) e/ou policloreto de vinila (PVC) através da separação dos MP por densidade, fazendo uso de soluções salinas e alcoólicas com densidades variando entre 0,91 e 1,11 g/ $\mathrm{cm}^{3}$ (Manrich et al., 1997; Spinacé \& Paoli, 2005).

Simultaneamente, foram coletadas, também em triplicatas, as amostras de água superficial junto aos pontos de coleta dos MP com o intuito de avaliar a qualidade da água e avaliar possíveis correlações entre a frequência de MP e a condição de qualidade a cada ponto amostral. Os parâmetros analisados de acordo com o Standard Methods for the Examination of Water and Wastewater (American Public Health Association, 2012) foram: potencial hidrogeniônico (pH), condutividade elétrica (CE), temperatura, cloretos, salinidade, ortofosfato, nitrogênio amoniacal, clorofila a, turbidez, oxigênio dissolvido (OD), demanda bioquímica de oxigênio no período de 5 dias $\left(\mathrm{DBO}_{5,20}\right)$, sólidos totais, sólidos sedimentáveis, sólidos suspensos e sólidos totais dissolvidos. Todas as análises foram realizadas no Laboratório de Análise de Águas e Sedimentos do Centro de Estudos Costeiros, Limnológicos e Marinhos (Ceclimar) da Universidade Federal do Rio Grande do Sul (Ufrgs), localizado às margens do ETA. Para avaliar um possível efeito de diluição também foram analisados os volumes de chuvas 7, 15 e 30 dias anteriores aos dias de coleta. Esses dados são provenientes do Instituto Nacional de Meteorologia, estação meteorológica de Tramandaí.

Para realizar a análise estatística dos dados foi utilizado o programa PAST (Paleontological Statistics software package for education and data analysis) version 2.08b (Hammer et al., 2001). Foram realizados testes de correlação de Pearson (r) com nível de significância de 1\% onde avaliaramse as correlações muito fortes $(|0,9|<r<|1|)$ e fortes $(|0,6|<r \leq|0,9|)$. Também, uma análise multivariada foi aplicada aos dados físicos e químicos da água e de contaminação por MP em cada um dos pontos. Foi realizada a análise hierárquica de cluster (Hierarchical Cluster Analýsis - HCA) e a análise de principais componentes (Principal Components Analysis - PCA).

\section{RESULTADOS E DISCUSSÕES}

\subsection{Análise quantitativa de MP}

A distribuição quantitativa de MP coletados, a profundidade e velocidade da água nos respectivos pontos de coleta estão apresentados na Tabela 1. Esta informação possibilita relacionar a localização dos MP com o impacto antropogênico por ocupação e a influência da hidrodinâmica sobre os mesmos.

A amostragem na Laguna do Armazém evidenciou ocorrência de MP. 0 ponto 1 apresentou o menor número de $\mathrm{MP}, 6$ und/100 $\mathrm{m}^{3}$, o que pode ser explicado principalmente pela ausência de atividade antrópica em áreas adjacentes (Figura 1) e pela sua proximidade com o Arroio Camarão, que é uma fonte de água mais doce e limpa que pode estar entrando na laguna e causando algum efeito de diluição. Além disso, o ponto 1 apresenta velocidade de água baixo (0,39 m/s) e maior profundidade $(0,75 \mathrm{~m})$, podendo haver sedimentação de MP.

Os pontos 2, 3 e 5 apresentaram 54,9; 33,7 e 43,1 und/100 $\mathrm{m}^{3}$ de MP, respectivamente. Esses pontos apresentam uma área de circulação preferencial das águas, que também é suscetível a ventos, aumentando a dispersão de MP nesses locais. 
Tabela 1. Distribuição de MP, profundidade e velocidade da água nos pontos amostrados nas lagunas Tramandaí e Armazém, Litoral Norte do RS.

\begin{tabular}{|c|c|c|c|c|}
\hline Ponto & Laguna & MP (und/100 $\mathrm{m}^{3}$ ) & Profundidade (m) & $\begin{array}{l}\text { Velocidade da água } \\
\qquad(\mathrm{m} / \mathrm{s})\end{array}$ \\
\hline 1 & \multirow{5}{*}{ Armazém } & 6,0 & 0,76 & 0,39 \\
\hline 2 & & 54,9 & 0,56 & 0,36 \\
\hline 3 & & 33,7 & 1,15 & 0,46 \\
\hline 4 & & 102,4 & 0,5 & 0,51 \\
\hline 5 & & 43,1 & 1,0 & 0,7 \\
\hline 6 & \multirow{6}{*}{ Tramandaí } & 50,8 & 0,7 & 0,48 \\
\hline 7 & & 41,3 & 1,5 & 0,49 \\
\hline 8 & & 31,6 & 1,25 & 0,52 \\
\hline 9 & & 31,9 & 1,5 & 0,42 \\
\hline 10 & & 12,0 & 1,0 & 0,78 \\
\hline 11 & & 57,3 & 1,10 & 0,45 \\
\hline
\end{tabular}

O ponto 4 apresentou a maior ocorrência de microplásticos, sendo 102,4 und/100 $\mathrm{m}^{3}$, essa região é a mais próxima a residências e comércios da Laguna Armazém, como pode ser observado na Figura 1. Outros estudos (Bertoldi et al., 2021; Wang et al., 2018) também observaram um relacionamento entre centros urbanos e distribuição microplástica onde a concentração de partículas de polímero foi maior em locais junto às cidades. Além disso, o ponto 4 possui a menor coluna d'água $(0,5 \mathrm{~m})$ da Laguna e pouca circulação da água $(0,51 \mathrm{~m} / \mathrm{s})$, fatores que podem justificar o maior número de MP registrados. De acordo com a literatura, a circulação de água é um fator que controla muito distribuição de MP, uma vez que um rápido fluxo acelera o transporte de e provoca espalhamento dos mesmos (Waldschläger et al., 2020).

A amostragem na Laguna Tramandaí exibiu ocorrência de MP sendo que os pontos 6 e 11 apresentaram um maior número de $\mathrm{MP}$, com 50,8 e 57,3 und/100 $\mathrm{m}^{3}$, respectivamente. Essa distribuição pode ser explicada por estes pontos estarem mais próximos da conurbação urbana do litoral (Figura 1, ponto 11) e também por ser o ponto de encontro do Rio Tramandaí com o estuário, onde podem chegar vários MP oriundos do subsistema norte, que fica a montante da Laguna Tramandaí (Castro \& Rocha, 2016) situação essa que pode ter colaborado para esses resultados terem demonstrado valor mais abundante. Já o ponto 6, como pode ser observado na Figura 1, tem influência da saída de água da Laguna Armazém. Esses dois pontos são também os que possuem uma maior influência marinha, sendo os pontos mais vulneráveis para receber MP da costa por conta da ocupação da área e da sua localização.

No ponto 10, houve um menor número de MP encontrados, sendo 12 und/100 $\mathrm{m}^{3}$, possivelmente em função da hidrodinâmica deste ponto, onde a circulação de água é mais rápida apresentando uma velocidade da água de $0,78 \mathrm{~m} / \mathrm{s}$, provavelmente, isso dificulta a permanência dos MP nestes locais. A circulação de água sendo menor no meio da laguna e maior nos pontos mais profundos dela, faz com que os pontos 7, 8 e 9 possuam um acúmulo maior deMP. Estes pontos possuem uma alta hidrodinâmica, sem ocorrência de atividade antrópica no seu entorno e a quantidade de seus MP é similar entre si, sendo elas, 41,3; 31,6 e 31,9 und/100 m³ respectivamente.

A amostragem de MP em águas ainda não está padronizada no mundo (Bertoldi et al., 2021). No entanto, comparamos os resultados da concentração média de microplásticos no ETA, com outros estudos em áreas estuarinas com metodologia semelhante (Tabela 2). De acordo com os dados reportados, a concentração encontrada apresentou-se semelhante a obtida no estudo do estuário Timonha-Ubatuba (Garcia et al., 2020) na divisa dos Estados do Ceará e Piauí/Brasil, que apresenta a maior e mais bem conservada área de manguezal remanescente do Ceará, onde $92 \%$ da comunidade é composto por pescadores artesanais (Pereira \& Rocha, 2015). Diante do exposto, é importante destacar que os MP têm sido encontrados mesmo em áreas mais bem preservadas como do estuário Timonha-Ubatuba, indicando que esses materiais podem ter uma origem mais distante do ponto de onde foram amostrados. 
Tabela 2 - Compilação de resultados obtidos para MP neste e em outros estudos similares.

\begin{tabular}{|c|c|c|c|}
\hline Referência & Localização & Método de amostragem & $\begin{array}{c}\text { Concentração } \\
\text { média } \\
\left.\text { (und } / 100 \mathrm{~m}^{3}\right)\end{array}$ \\
\hline Este estudo & $\begin{array}{l}\text { Estuário Tramandaí - Armazém, } \\
\text { RS/Brasil }\end{array}$ & $\begin{array}{l}\text { Rede de plâncton, } \\
\text { malha } 300 \mu \mathrm{m}\end{array}$ & 42,30 \\
\hline $\begin{array}{l}\text { Carpenter \& Smith } \\
\text { Junior (1972) }\end{array}$ & $\begin{array}{c}\text { Estuário de Long Island, } \\
\text { CT/EUA }\end{array}$ & $\begin{array}{l}\text { Rede de plâncton, } \\
\text { malha } 333 \mu \mathrm{m}\end{array}$ & 27,5 \\
\hline Lima et al. (2014) & Estuário de Goiânia, PE/Brasil & $\begin{array}{l}\text { Rede de plâncton, } \\
\text { malha } 300 \mu \mathrm{m}\end{array}$ & 0,26 \\
\hline Garcia et al. (2020) & $\begin{array}{l}\text { Estuário Timonha-Ubatuba, } \\
\text { divisa dos Estados do Ceará e } \\
\text { Piauí/Brasil }\end{array}$ & $\begin{array}{l}\text { Rede de plâncton, } \\
\text { malha } 300 \mu \mathrm{m}\end{array}$ & $45-66$ \\
\hline Garcia et al. (2020) & Estuário Jaguaribe, CE/Brasil & $\begin{array}{l}\text { Rede de plâncton, } \\
\text { malha } 300 \mu \mathrm{m}\end{array}$ & $0,2-2,3$ \\
\hline Olivatto (2016) & $\begin{array}{l}\text { Porção oeste da Baía de } \\
\text { Guanabara, RJ/Brasil }\end{array}$ & $\begin{array}{l}\text { Rede de plâncton, } \\
\text { malha } 300 \mu \mathrm{m}\end{array}$ & 1071 \\
\hline
\end{tabular}

Porém, sabe-se que estuários são ambientes vulneráveis e dinâmicos, sendo que muitos deles já se encontram modificados devido à poluição causada pelo aumento da urbanização e de atividades industriais (Vilela et al., 2014). A título de exemplo do exposto, a Baía de Guanabara no Rio de Janeiro é considerada um dos ecossistemas mais poluídos do Brasil (Vilela et al., 2014) e sofreu modificações advindas de atividade antrópica, escoamento urbano, agrícola e industrial nos últimos 100 anos (Baptista Neto \& Fonseca, 2011), constatação que se consolida segundo Castro \& Rocha (2016) e Olivatto (2016) através das concentrações expressivas de poluição microplástica diagnosticada em seus estudos.

\subsection{Análise qualitativa dos MPs}

A existência de MP em água pode ser atribuída a muitas fontes e fatores, a discussão das origens é um desafio, uma vez que seu mecanismo de difusão e transporte é extremamente complexo (Bertoldi et al., 2021). No entanto, analisando diretamente os percentuais encontrados, no sistema estuarino Tramandaí-Armazém, o PEAD e o PET/PVC foram os polímeros mais abundantes coletados, seguidos pelo PEBD (Figura 2). A contaminação por estes polímeros pode estar diretamente relacionada ao uso e ocupação do estuário por atividade pesqueira e pela urbanização do local, já que polietileno (subdividido em PEAD e PEBD) é o principal componentes das redes, linhas de pesca e de sacolas plásticas e embalagens (Vogelmann et al., 2019; Wang et al., 2018), enquanto PET/PVC, estão relacionados com boias e tubulações (PVC) (Ding et al., 2021) e embalagens de bebidas (Kankanige \& Babel, 2020).

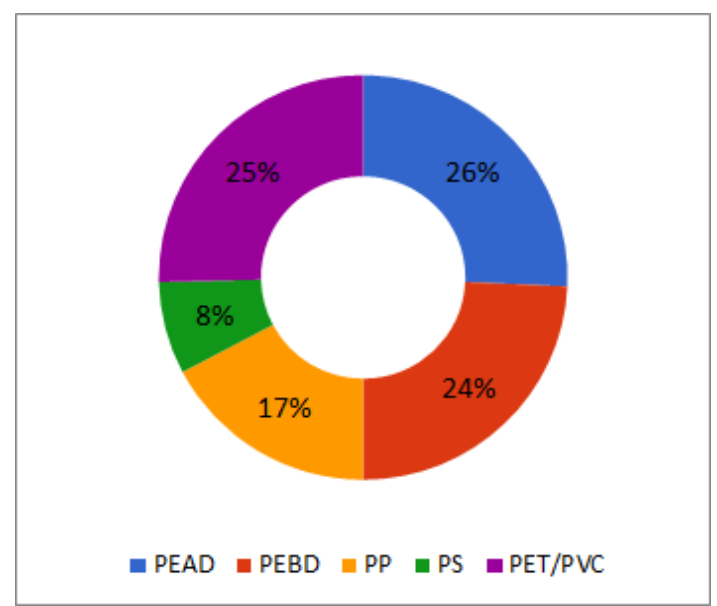

Figura 2. Composição polimérica dos microplásticos obtidos no Sistema Estuarino Tramandaí-Armazém. 
A Figura 3 ilustra os percentuais obtidos para cada classe polimérica nas lagunas Armazém e Tramandaí, sendo importante evidenciar que o PEAD, PEBD, PP, PVC e PS representam, aproximadamente, $90 \%$ da produção global de polímeros e incluem-se no grupo dos commodities (polímeros convencionais), que se caracterizam por baixo custo e elevada produção (Andrady, 2011).

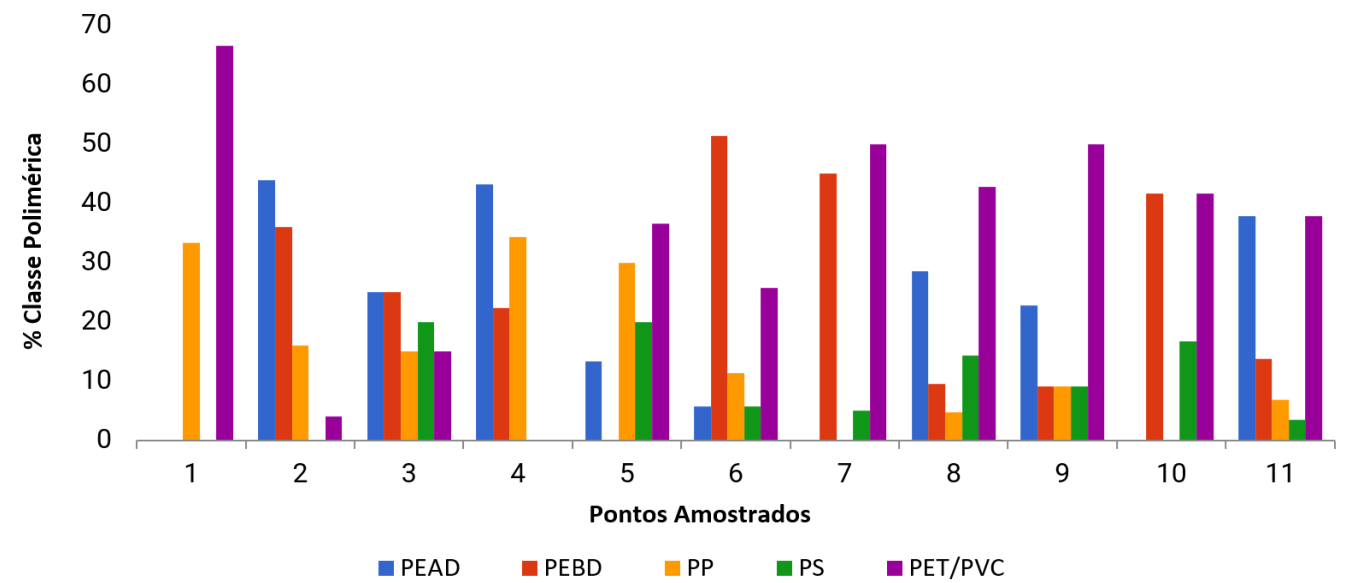

Figura 3. Composição polimérica, por pontos, dos microplásticos coletados no Sistema Estuarino TramandaíArmazém .

Do total de MP coletados na Laguna do Armazém (pontos 1-5), o polietileno apresentou maior ocorrência (54,9\%), estando subdividido em PEAD (34,4\%) e PEBD (20,6\%). Utilizado na fabricação de embalagens de alimentos, produtos de higiene e como principal fonte de produção de sacolas plásticas (Associação Brasileira da Indústria do Plástico, 2017), suas características e aplicações explicam a quantidade majoritária entre os microplásticos coletados e entre os pontos amostrados. Além disso, a intensa atividade de pesca que ocorre na ETA pode contribuir para isto, visto ser o principal componente de redes e linhas de pesca, sendo o polietileno ocorrente em quatro dos cinco pontos amostrados (Wang et al., 2018).

Representando 28,3\% de todos os MP coletados na Laguna dos fragmentos plásticos coletados, o PP foi o segundo polímero de maior ocorrência na Laguna Armazém. Possui resistência química e a altas temperaturas, sendo utilizado em embalagens de alimentos, garrafas, autopeças e fibras (Associação Brasileira da Indústria do Plástico, 2017), ou seja, amplo espectro de aplicação e, potencialmente, de distribuição no ambiente.

Polipropileno e polietileno também compreenderam a maioria (98\%) dos tipos de polímeros observados em amostras coletadas no Lagoa Guaíba, sul do Brasil (Bertoldi, et al., 2021). A predominância desses polímeros pode ainda ser atribuída às baixas densidades (PP $0.92 \mathrm{~g} / \mathrm{cm}^{3}$, PEAD/PEBD 0.90-0.97 $\mathrm{g} / \mathrm{cm}^{3}$ ) que estes materiais possuem. São geralmente flutuantes, facilitando seu transporte na superfície da coluna d'água, o que contribui para sua ampla distribuição (Bertoldi et al., 2021; Wang et al., 2018).

O maior percentual de PET/PVC foi encontrado no ponto 1 (\%??), local com menor quantidade de MP do estuário. No entanto, PET/PVC, juntamente com o PS, registraram os menores percentuais na Laguna Armazém, 9,2\% e 7,1\%, respectivamente. Ambos são resistentes à degradação e utilizados desde a produção de embalagens até produtos de construção civil, esta última sendo uma das atividades mais intensamente desenvolvidas na região.

Na Laguna Tramandaí (pontos 6-11), do total de MP coletados na laguna, o polietileno também apresentou uma maior ocorrência, com cerca de $46 \%$, sendo subdividido em PEAD $(17,3 \%)$ e PEBD $(28,8 \%)$. A predominância de polietileno como já citado acima, é consistente com sua enorme produção global e amplo uso na vida moderna, como garrafas, sacos, filmes, tampas e recipientes de plástico (PlasticsEurope, 2016). Seu uso também está relacionado à intensa atividade pesqueira na região, já que este é o principal componente das redes e linhas de pesca. Portanto, essas partículas de PEAD e PEBD são, provavelmente, MP secundários. Além disso, devido à densidade mais baixa, esses polímeros geralmente flutuam e podem ser facilmente transportados pela água, o que também pode favorecer a ampla distribuição desse material (Wang et al., 2018). 
Representando 39,6\% dos fragmentos coletados, o PET/PVC foi a segunda classe de polímeros de maior ocorrência na Laguna Tramandaí. O PET é muito utilizado em garrafas de bebidas, embalagem de alimentos, de cosméticos e de remédios, enquanto o PVC possui uma aplicação em filmes, laminados, brinquedos, embalagens para remédios e tubulação para água e esgoto (Associação Brasileira da Indústria do Plástico, 2017). A grande quantidade de PET/PVC encontrados, pode se relacionar com a ampla circulação de embarcações neste local. Ainda que de pequeno porte, essas embarcações podem apresentar a degradação da sua pintura, que consequentemente libera partículas plásticas no meio aquático.

O PS e o PP foram os polímeros que registraram os menores percentuais na Laguna Tramandaí, sendo 7,9\% e 6,5\%, respectivamente. São utilizados em embalagens industriais, fios e cabos, frascos, autopeças, tampas, potes, fraldas e seringas descartáveis (Associação Brasileira da Indústria do Plástico, 2017).

\subsection{Dados limnológicos e sua relação com o MP}

Os dados físicos e químicos determinados a partir das amostras coletadas concomitantes às amostras de MP são apresentados no Quadro 1.

Quadro 1 - Variáveis físicas e químicas das amostras de águas coletadas concomitantes às coletas de MP nas lagunas Tramandaí e Armazém.

\begin{tabular}{|c|c|c|c|c|c|c|c|c|c|c|c|c|}
\hline \multirow{3}{*}{ Variáveis } & \multirow{3}{*}{ Unidades } & \multicolumn{11}{|c|}{ Pontos } \\
\hline & & \multicolumn{5}{|c|}{ Verão } & \multicolumn{6}{|c|}{ Inverno } \\
\hline & & 1 & 2 & 3 & 4 & 5 & 6 & 7 & 8 & 9 & 10 & 11 \\
\hline Temp. da água & ${ }^{\circ} \mathrm{C}$ & 31,0 & 30,0 & 30,0 & 30,0 & 29,0 & 17,5 & 17,5 & 17,2 & 17,2 & 17,2 & 16,8 \\
\hline Condutividade & $\mathrm{uS} / \mathrm{cm}$ & 12,57 & 11,77 & 11,92 & 11,45 & 10,71 & 3,08 & 3,01 & 3,55 & 0,08 & 3,28 & 0,06 \\
\hline Transparência & $\mathrm{cm}$ & 31 & 48 & 64 & 38 & 35 & 30 & 65 & 50 & 80 & 50 & 70 \\
\hline Clorofila a & $\mathrm{ug} / \mathrm{L}$ & 39,6 & 10,2 & ND & 4,5 & 1,1 & 18,1 & ND & 30,5 & 6,8 & 67,8 & 5,7 \\
\hline $\mathrm{DBO}_{5}$ & $\mathrm{mg} / \mathrm{L}$ & 3,07 & 3,07 & 2,63 & 3,13 & 2,49 & 1,40 & 1,99 & 1,20 & 1,69 & 0,80 & 1,60 \\
\hline $\begin{array}{l}\text { Nitrogênio } \\
\text { amoniacal }\end{array}$ & $\mathrm{mg} / \mathrm{L}$ & 0,02 & ND & ND & ND & ND & 0,07 & 0,02 & 0,02 & 0,02 & 0,02 & 0,02 \\
\hline Ortofosfato & $\mathrm{mg} / \mathrm{L}$ & 0,02 & ND & ND & ND & ND & 0,07 & 0,02 & 0,02 & 0,02 & 0,02 & 0,02 \\
\hline $\begin{array}{c}\text { Oxigênio } \\
\text { dissolvido }\end{array}$ & $\mathrm{mg} / \mathrm{L}$ & 6,91 & 8,06 & 6,35 & 8,06 & 7,36 & 7,39 & 8,80 & 8,10 & 8,45 & 8,45 & 7,92 \\
\hline $\mathrm{pH}$ & - & 7,78 & 7,93 & 7,92 & 7,89 & 7,83 & 7,31 & 7,41 & 7,34 & 7,21 & 7,46 & 7,20 \\
\hline Cloretos & $\mathrm{mg} / \mathrm{L}$ & 10497 & 9874 & 9597 & 9694 & 9196 & 1593 & 1593 & 1731 & 26 & 1593 & 2 \\
\hline $\begin{array}{c}\text { Sól. } \\
\text { Sedimentáveis } \\
\end{array}$ & $\mathrm{mg} / \mathrm{L}$ & ND & ND & ND & ND & ND & 0,3 & ND & ND & ND & ND & ND \\
\hline Sól. Totais & $\mathrm{mg} / \mathrm{L}$ & 11941 & 11519 & 11788 & 11096 & 10075 & 2528 & 2894 & 2941 & 115 & 2607 & 91 \\
\hline $\begin{array}{c}\text { Sól. Tot. } \\
\text { dissolvidos }\end{array}$ & $\mathrm{mg} / \mathrm{L}$ & 11870 & 11438 & 11732 & 11033 & 10022 & 2459 & 2891 & 2939 & 105 & 2591 & 75 \\
\hline Sól. Suspensos & $\mathrm{mg} / \mathrm{L}$ & 71 & 81 & 56 & 63 & 53 & 69 & 3 & 2 & 10 & 16 & 16 \\
\hline Turbidez & NTU & 21,9 & 8,5 & 10,1 & 10,9 & 12,4 & 228,2 & 120,7 & 7,3 & 39,3 & 149,2 & 52,0 \\
\hline
\end{tabular}

Muitos resíduos plásticos podem ter sua origem no escoamento superficial direto e, desta maneira, períodos chuvosos poderiam contribuir para maiores concentrações. Dessa forma, os volumes de chuvas 7, 15 e 30 dias anteriores aos dias de coleta estão sendo apresentados na Tabela 3. Considerando o tamanho da bacia, é difícil predizer em qual período de tempo seria correto considerar, mas em linhas gerais, nos 7 dias que antecederam as coletas, ocorreu uma precipitação nula ou muito baixa. A diferença de precipitação foi inexistente ou pequena entre os períodos, que pouco justificaria a distribuição dos MP. 
Tabela 3 - Chuvas acumuladas 7, 15 e 30 dias antes das coletas.

\begin{tabular}{c|c|c}
\hline \multirow{2}{*}{$\begin{array}{c}\text { Número de dias que antecedem } \boldsymbol{a} \\
\text { coleta }\end{array}$} & \multicolumn{2}{c}{ Precipitação acumulada (mm) } \\
\cline { 2 - 3 } & 0 & JuI \\
\hline 7 & 0 & 0,6 \\
\hline 15 & 26,6 & 16,8 \\
\hline 30 & 39,4 \\
\hline
\end{tabular}

Fonte: Instituto Nacional de Meteorologia (2020).

Como a amostragem se dividiu em duas estações opostas, verão e inverno, as variáveis que sofrem influência climática direta mostraram nítida diferença, sendo elas a temperatura das águas, o oxigênio dissolvido e a demanda bioquímica de oxigênio.

Entretanto, entre outras variáveis, estas duas últimas podem ser influenciadas pela fator poluição uma vez que a elevada concentração de matéria orgânica consome o oxigênio das águas quando da sua degradação. Em casos como este, é necessário monitorar por período mais longo o recurso hídrico para isolar a influência dos diferentes fatores, inclusive porque as concentrações de OD variaram entre 6,35 e 8,80 mg/L (Quadro 1), com média para o janeiro e julho de 2019, respectivamente, de 7,36 \pm 0,74 e $8,18 \pm 0,5 \mathrm{mg} / \mathrm{L}$. A DBO 5 também apresentou valores médios diferentes, sendo 2,88 $\pm 0,3 \mathrm{mg} / \mathrm{L}$ em Jan/19 e 1,45 $\pm 0,42 \mathrm{mg} / \mathrm{L}$ em Jul/19, variando entre 0,80 e 3,13 mg/L de oxigênio consumido dentre as amostragens executadas.

Outras variáveis mostraram influência marinha, sendo elas condutividade, $\mathrm{pH}$, teor de cloretos, os diferentes indicadores de sólidos e turbidez (Esteves, 2011). Esta influência já era esperada, entretanto em alguns casos ela pode esconder o efeito da poluição nas águas, e como ela age de forma diferente entre as lagunas Tramandaí e Armazém, em vista da hidrodinâmica, dificultam o processo de comparação.

Além dos teores de $\mathrm{OD}$ e $\mathrm{DBO}_{5}$, as concentrações de clorofila a e nutrientes são aquelas características em que mais se espera efeito por poluição por esgotos domésticos (Castro, 2019). Embora tenha se observado diferenças no teor de clorofila a entre os pontos amostrados (Quadro 1), para nitrogênio amoniacal e ortofosfato isso não foi verificado, estando as concentrações muito próximas da faixa não detectável.

Considerando isso, os dados da qualidade da água e da quantidade total de MP foram submetidos a testes de correlação, onde a influência marinha e climática ficou confirmada, por exemplo, com correlações significativas $(\mathrm{p}<0,01)$ já esperadas entre temperaturas da água e do ar, $\mathrm{DBO}_{5}$, condutividade, cloretos, pH, sólidos totais e sólidos totais dissolvidos (Quadro 2).

0 teor de cloretos, devido à água salobra mostrou correlação positiva significativa forte $(\mathrm{r}=0,89)$ também com a $\mathrm{DBO}_{5}$, indicando que a demanda é maior em águas mais salobras. Entretanto, esta relação é fomentada pelo período amostral, já que as amostras mais salobras são da Laguna Armazém, coletada em Jan/19, período de aceleração do consumo de oxigênio. Além disso, houve correlação significativa positiva muito forte $(\mathrm{r}=0,98)$ entre os nutrientes ortofosfato e nitrogênio amoniacal, e destes com o teor de sólidos sedimentáveis ( $\mathrm{r}=0,9$ e $\mathrm{r}=087$, respectivamente), indicando que parte expressiva dos nutrientes está contida nas partículas sedimentáveis das referidas amostras. A relação inversa entre profundidade e sólidos suspensos $(r=0,84)$ também ficou caracterizada de forma significativa.

Os dados físicos e químicos também foram avaliados buscando possíveis correlações com a distribuição categórica dos MP, mas nenhuma relação importante $\left(r^{2}<0,6\right)$ foi verificada. Variáveis habitualmente influenciadas por efeito de poluição, como anteriormente mencionadas (OD, DBO5, nutrientes e clorofila a) eram aquelas esperadas potenciais correlações. Entretanto, baixas concentrações, variações pouco significativas entre pontos, ou mesmo variáveis influenciadas paralelamente por condições climáticas dificultam tal percepção. 0 cenário do ETA é complexo em virtude de forçantes múltiplas, que acabam por mascarar causa e efeito diretos; inclusive havendo efeitos potencializados pela concorrência de mais de uma causa, neste caso, a poluição, somada ao veraneio e a ocupação sazonal implicada, entre outros.

Ainda, tratando-se de uma avaliação preliminar para região, não foram considerados outros parâmetros poluentes, como aqueles emergentes, agroquímicos ou metais. Isso se baseia na complexidade, custo e nas possiveis fontes menos presentes na região comparados com outras localidades. Considerando não serem relatados na literatura estudos que comparem a qualidade física e química das águas com a presença de MP, iniciar por uma avaliação que pondere sobre causas mais 
genéricas traz um panorama inicial justificável. Somado a isso, diversos estudos relacionam a presença de MP com densidade populacional e consequente presença de esgotos sanitários (Bertoldi et al. 2021; Carvalho, et al., 2021), situação similar a observada na área de estudo proposta.

Quadro 2 - Correlação de Pearson ( $r)$ com a respectiva significância $(\mathrm{p}<0,01)$ entre os teores de microplásticos e as variáveis físicas e químicas nas lagunas Tramandaí e Armazém.

\begin{tabular}{|c|c|c|c|c|c|c|c|c|c|c|c|c|c|c|c|c|c|c|}
\hline & LLag & PEAD & PPP & MMP & TTar & TTag & CCon & PPro & DDBO & NNam & OOrt & ppH & CClo & SSSe & SST & SSTD & SSSu & TTur \\
\hline LLag & 1 & & & & & & & & & & & & & & & & & \\
\hline PPEAD & & 1 & & & & & & & & & & & & & & & & \\
\hline PPP & & 00,91 & 1 & & & & & & & & & & & & & & & \\
\hline MMP & & 0,87 & 0,91 & 1 & & & & & & & & & & & & & & \\
\hline Tar & $-0,86$ & & & & 1 & & & & & & & & & & & & & \\
\hline TTag & $-0,99$ & & & & 00,87 & 1 & & & & & & & & & & & & \\
\hline CCon & $-0,97$ & & & & 00,81 & 0,98 & 1 & & & & & & & & & & & \\
\hline PPro & & & & & & & & 1 & & & & & & & & & & \\
\hline DDBO & $-0,91$ & & & & 00,82 & 0,92 & 0,86 & & 1 & & & & & & & & & \\
\hline NNam & & & & & & & & & & 1 & & & & & & & & \\
\hline OOrt & & & & & & & & & & 0,98 & 1 & & & & & & & \\
\hline ppH & $-0,96$ & & & & & 0,96 & 0,98 & & 0,83 & & & 1 & & & & & & \\
\hline CClo & $-0,99$ & & & & 00,85 & 0,99 & 0,99 & & 0,89 & & & 0,97 & 1 & & & & & \\
\hline SSSe & & & & & & & & & & 0,90 & 0,87 & & & 1 & & & & \\
\hline SST & $-0,98$ & & & & 0,82 & 0,98 & 0,99 & & 0,87 & & & 0,98 & 0,99 & & 1 & & & \\
\hline SSTD & $-0,98$ & & & & 0,82 & 0,98 & 0,99 & & 0,87 & & & 0,98 & 0,99 & & 0,99 & 1 & & \\
\hline SSSu & & & & & & & & $-0,84$ & & & & & & & & & 1 & \\
\hline TTur & & & & & & & & & & 0,82 & 0,83 & & & & & & 1 \\
\hline
\end{tabular}

Legenda: Lag = lagoa; MP = no total de MP; Tar = temperatura do ar; Tag = temperatura da água; Con = condutividade; Pro = profundidade; Nam = nitrogênio amoniacal; Ort = ortofosfato; $\mathrm{Clo}=$ cloretos; $\mathrm{SSe}=$ sólidos sedimentáveis; $\mathrm{ST}=$ sólidos totais; $\mathrm{STD}=$ sólidos totais dissolvidos; SSu = sólidos em suspensão; Tur = turbidez.

Vale ressaltar que o tamanho e a densidade dos materiais plásticos atuam diretamente na flutuabilidade, sua posição na coluna d'água e a interação com a biota (Browne et al., 2010; Wright et al., 2013; Andrady, 2011), trazendo outros tipos de efeitos. Alterações na sua densidade, ocasionados pela fixação de filmes biológicos, degradação e fragmentação também são fatores que modificam a distribuição do plástico na coluna de água (Avio et al., 2016).

Outras correlações encontradas e apresentadas no Quadro 2 foram a do PEAD com PP $(r=0,91$; muito forte), PEAD com o número geral de MP ( $\mathrm{r}=0,87$, forte), e do PP com o número total de MP $(r=0,91$, muito forte). Estas relações indicam que tanto PEAD como PP apresentam uma distribuição que acompanha a distribuição geral do MP, bem como, possivelmente, a fonte de PEAD e de PP sejam as mesmas, ou que sejam geradas por produtos semelhantes ou de matéria-prima combinada.

Uma análise multivariada (Figura 4) foi aplicada aos dados físicos e químicos da água e de contaminação por MP em cada um dos pontos.

Tanto a análise hierárquica de cluster (HCA - Figura 4a), quanto a análise de principais componentes (PCA - Figura 4b) evidenciam a formação de 3 grupos:

i) ponto 4 , destacado por seu valor mais expressivo de $\mathrm{DBO}_{5}$ e o número mais elevado em MP de forma geral (24\%) e nos grupos PEAD, PEBD e PP;

ii) pontos 1, 2, 3 e 5, referentes à Laguna Armazém;

iii) pontos 6 a 11, referentes à Laguna Tramandaí.

Os dois últimos grupos foram separados, quase que exclusivamente, com base nas características físicas e químicas das águas, que neste caso denotam lagoa e estação. Quanto ao destaque do ponto 4 e a relação observada, possivelmente a mesma pode ser evidência importante da relação contaminação por MP e despejo de esgotos.

Os componentes 1 e 2 avaliados pela PCA respondem por 41 e 19\%, respectivamente. 0 componente 1 é composto pelas variáveis temperatura da água, condutividade, $\mathrm{DBO}_{5}, \mathrm{pH}$, cloretos e sólidos totais dissolvidos, enquanto que o componente 2 é composto mais expressivamente por PEBD 
e MP em geral. Ou seja, eixos bem definidos pelas características físicas e químicas e pelas características de MP.

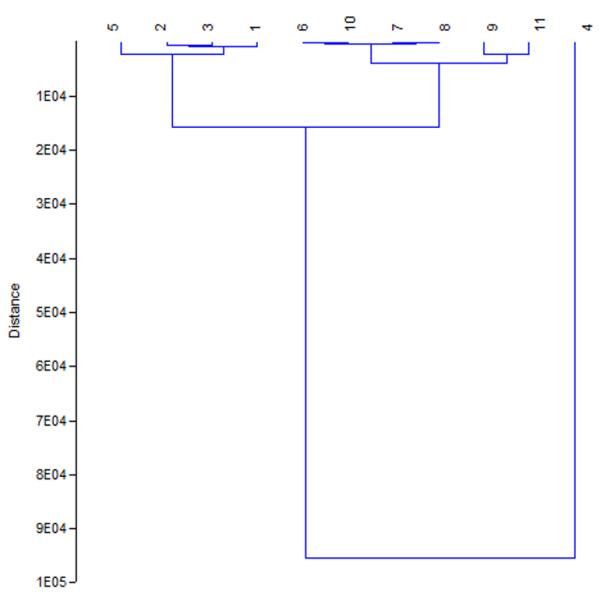

b)

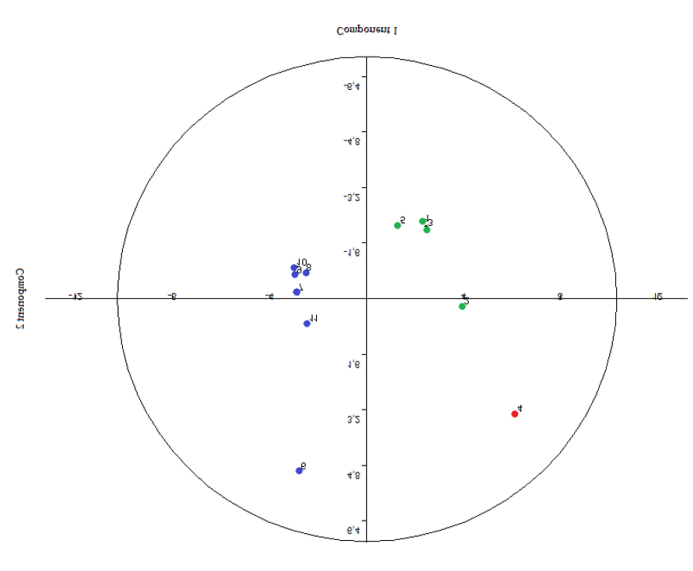

Figura 4 - Análise multivariada dos dados físicos e químicos da água e de contaminação por MP nos pontos: a) Hierarchical Cluster Anayýsis, HCA; b) Principal Components Analysis, PCA

Avaliando de forma multivariada apenas os dados de MP, fica evidente a separação por quantitativo dos diferentes tipos de MP, destacando-se a amostra do ponto 4; seguido pelo grupo dos pontos 2, 3, 5, 7, 8, 9 e 11 com contaminação mediana de MP; enquanto os pontos 1, 6 e 10 apresentam os menores valores.

Por fim, avaliando apenas os dados físicos químicos das águas, há a formação de dois agrupamentos, praticamente a replicação dos agrupamentos formados quando a análise era geral, combinando dados físicos e químicos da água e de MP. O destaque nesta avaliação é a formação dos eixos da PCA, sendo eles: componente 1, explicando 48\% das características amostrais, com destaque para as variáveis temperatura da água, condutividade, $\mathrm{DBO}_{5}, \mathrm{pH}$, cloretos e sólidos totais dissolvidos, todos potencialmente mais vinculados à questão da cunha salina; e o componente 2, explicando 20\% das características amostras, destacando as variáveis potencialmente vinculadas à água doce aos efeitos de poluição e como nitrogênio amoniacal, ortofosfato e sólidos sedimentáveis.

\section{COMENTÁRIOS FINAIS}

Os dados obtidos neste estudo compõem um panorama pioneiro sobre a contaminação por MP na região do Litoral Norte do RS. Os resultados evidenciam ser uma área que já apresenta contaminação, destacando-se o PE, o PP e o PET/PVC, estreitamente vinculados às atividades desenvolvidas na região, como a pesca e a ocupação urbana em si.

A análise dos dados de qualidade não deixou clara a vinculação de poluição das águas com a presença de MP. No entanto, o ponto 4, apresentou na análise multivariada seu valor mais expressivo de $\mathrm{DBO}_{5}$ e o número mais elevado em MP de forma geral. Possivelmente esse resultado pode ser evidência importante da relação contaminação por MP e despejo de esgotos. Em geral, a poluição das águas por esgotos e também por MP pode estar ligada a variação da densidade populacional. Também se faz importante considerar efeitos de poluição difusa a fim de se verificar a influência de efeitos de diluição para poder explicar, por exemplo, os maiores índices de DBO, sólidos suspensos e cloretos em uma laguna do que em outra.

A busca por uma avaliação espacial em detrimento da influência temporal justificou as amostragens em duas estações distintas. Entretanto, não é possível eliminar completamente a influência vinculada à temporalidade sem uma amostragem comparativa entre estações. Possivelmente, a reprodução de avaliações por um número maior de períodos evidenciaria quais os componentes que mais influenciam sobre a poluição por MP. Embora haja a compreensão de que os MP são resultantes de plásticos sujeitos a efeitos ambientais e isso pode demandar muitos dias, meses ou até anos, não é possível fechar esta questão sem antes uma avaliação de mais longo período, que vai inclusive indicar o efeito, por exemplo, da ocupação massiva durante o veraneio. 
Ainda, é essencial que tais dados sejam levados à público para contribuir nas decisões regionais acerca da gestão e educação ambiental, visto que sem modificações no comportamento de uso e descarte este quadro deverá piorar rapidamente.

\section{AGRADECIMENTOS}

Os autores gostariam de agradecer ao CECLIMAR/CLN/UFRGS, pela oportunidade de utilizar o Laboratório de Análise de Águas e Sedimentos para a realização das análises, assim como a Universidade Estadual do Rio Grande do Sul pelo apoio recebido, e a todos colaboradores do projeto.

\section{REFERÊNCIAS BIBLIOGRÁFICAS}

Almeida, L. E. S. B., Rosauro, N. M. L., \& Toldo Junior, E. E. (1997). Análise preliminar das marés na barra do Rio Tramandaí (RS - Brasil). In Anais do $12^{\circ}$ Simpósio Brasileiro de Recursos Hídricos (pp. 559-566). Vitória.

Alomar, C., Estarellas, F., \& Deudero, S. (2016). Microplastics in the Mediterranean Sea: deposition in coastal shallow sediments, spatial variation and preferential grain size. Marine Environmental Research, 115, 1-10. http://dx.doi.org/10.1016/j.marenvres.2016.01.005

American Public Health Association - APHA. American Water Works Association - AWWA. Water Environmental Federation - WEF. (2012). Standard methods for the examination of water and wastewater. Washington, D.C.: APHA.

Andrady, A. L. (2011). Microplastics in the marine environment. Marine Pollution Bulletin, 62(8), 1596-1605. http://dx.doi.org/10.1016/j.marpolbul.2011.05.030

Associação Brasileira da Indústria do Plástico - ABIPLAST. (2017). Recuperado em 26 de janeiro de 2021, de http://www.abiplast.org.br/wp-content/uploads/2019/03/Perfil-2017.pdf

Avio, C. G., Gorbi, S., \& Regoli, F. (2016). Plastics and microplastics in the oceans: from emerging pollutants to emerged threat. Marine Environmental Research, 128, 1-10. http://dx.doi.org/10.1016/j.marenvres.2016.05.012

Baptista Neto, J. A., \& Fonseca, E. M. (2011). Variação sazonal, espacial e composicional de lixo ao longo das praias da margem oriental da Baía de Guanabara (Rio de Janeiro) no período de 1999-2008. Revista de Gestão Costeira Integrada, 11(1), 31-39. http://dx.doi.org/10.5894/rgci189

Bertoldi, C., Lara, L. Z., Mizushima, F. A. L., Martins, F. C. G., Battisti, M. A., Hinrichs, R., \& Fernandes, A. N. (2021). First evidence of microplastic contamination in the freshwater of Lake Guaíba, Porto Alegre, Brazil. The Science of the Total Environment, 759, 143503. http://dx.doi.org/10.1016/j.scitotenv.2020.143503

Browne, M. A., Galloway, T. S., \& Thompson, R. C. (2010). Spatial patterns of plastic debris along estuarine shorelines. Environmental Science \& Technology, 44(9), 3404-3409. http://dx.doi.org/10.1021/es903784e

Carpenter, E. J., \& Smith Junior, K. L. (1972). Plastics on the Sargasso Sea. Surface Science, 175(4027), 1240-1241. http://dx.doi.org/10.1126/science.175.4027.1240

Carvalho, A. R., Garcia, F., Riem-Galliano, L., Tudesque, L., Albignac, M., ter Halle, A., \& Cucherousset, J. (2021). Urbanization and hydrological conditions drive the spatial and temporal variability of microplastic pollution in the Garonne River. The Science of the Total Environment, 769, 144479. http://dx.doi.org/10.1016/j.scitotenv.2020.144479

Castro, D. (2019). Ciclo das águas na bacia hidrográfica do Rio Tramandaí (Cap. 4, 176 p.). Porto Alegre: Via Sapiens.

Castro, D., \& Mello, R. (2013). Atlas Ambiental da Bacia Hidrográfica do rio Tramandaí (182 p.). Porto Alegre: Via Sapiens.

Castro, D., \& Rocha, C. M. (2016). Qualidade das águas na bacia hidrográfica do rio Tramandaí (172 p.). Porto Alegre: Via Sapiens.

Cole, M., Lindeque, P. K., Fileman, E., Clark, J., Lewis, C., Halsband, C., \& Galloway, T. S. (2016). Microplastics alter the properties and sinking rates of zooplankton faecal pellets. Environmental Science \& Technology, 50(6), 3239-3246. http://dx.doi.org/10.1021/acs.est.5b05905

Dillenburg, S.R. (1994). Laguna de Tramandaí: evolução geológica e aplicação da técnica de datação por termoluminescência na datação dos sedimentos lagunares (Tese de doutorado). Universidade Federal do Rio Grande do Sul, Porto Alegre. 
Ding, J., Sun, C., He, C., Li, J., Ju, P., \& Li, F. (2021). Microplastics in four bivalve species and basis for using bivalves as bioindicators of microplastic pollution. The Science of the Total Environment, 782(15), 146830. http://dx.doi.org/10.1016/j.scitotenv.2021.146830

Esteves, F. A. (2011). Fundamentos de limnologia (3. ed., 826 p.). Rio de Janeiro: Interciência.

Garcia, T. M., Campos, C. C., Mota, E. M. T., Santos, N. M. O., Campelo, R. P. de S., Prado, L. C. G., Junior, M. M., Soares, M. de 0. [2020]. Microplastics in subsurface waters of the western equatorial Atlantic (Brazil). Marine Pollution Bulletin, 150 (2020) 110705. https://doi.org/10.1016/j.marpolbul.2019.110705

Hammer, 0., Haper, D.A.T., Ryan, P.D. [2001].Paleontological Statistics software package for education and data analysis. Palaeontologia Electronica 4(1):9 pp.

Instituto Nacional de Meteorologia - INMET. (2020). Banco de dados meteorológicos: estação automática de Tramandaí. Ministério da Agricultura, Pecuária e Abastecimento. Recuperado em 26 de janeiro de 2021, de https://portal.inmet.gov.br/

Ivar do Sul, J. A., Spengler, A., \& Costa, M. F. (2009). Here, there and everywhere. Small plastic fragments and pellets on beaches of Fernando de Noronha (Equatorial Western Atlantic). Marine Pollution Bulletin, 58(8), 1236-1238. http://dx.doi.org/10.1016/j.marpolbul.2009.05.004

Jiang, J.-Q. (2018). Occurrence of microplastics and its pollution in the environment: a review. Sustainable Production and Consumption, 12, 16-23. http://dx.doi.org/10.1016/j.spc.2017.11.003

Joint Group of Experts on the Scientific Aspects of Marine Environmental Protection - GESAMP. (2015). Sources, fate and effects of microplastics in the marine environment: a global assessment (Reports Stud. GESAMP, Vol. 90, 96 p.). Recuperado em 26 de janeiro de 2021, de https://ec.europa.eu/environment/marine/goodenvironmental-status/descriptor-10/pdf/GESAMP_microplastics\%20full\%20study.pdf

Kankanige, D., \& Babel, S. (2020). Smaller-sized micro-plastics (MPs) contamination in single-use PETbottled water in Thailand. The Science of the Total Environment, 717(15), 137232. http://dx.doi.org/10.1016/j.scitotenv.2020.137232

Liddell, H. G., \& Scott, R. (1889). Plastikos. In An Intermediate Greek English Lexicon. Oxford: Clarendon Press, Ed. Perseus Digital Library.

Lima, A.R.A., Costa, M.F., Barletta, M., 2014. Distribution patterns of microplastics within the plankton of a tropical estuary. Environ. Res. 132, 146-55. doi:10.1016/j.envres.2014.03.031

Lippiatt, S., Opfer, S., \& Arthur, C. (2013). Marine Debris monitoring and assessment (NOAA Technical Memorandum NOS-OR\&R-46). Silver Spring. Recuperado em 26 de janeiro de 2021, de https://marinedebris.noaa.gov/sites/default/files/Lippiatt\%20et\%20al\%202013.pdf

Lozoya, J. P., Carranza, A., Lenzi, J., Machín, E., Mello, F. T., González, S., Hernández, D., Lacerot, G., Martínez, G., Scarabino, F., Sciandro, J., Vélez-Rubio, G., Burgues, F., Carrizo, D., Cedrés, F., Chocca, J., Álava, D., Jiménez, S., Leoni, V., Limongi, P., López, G., Olivera, Y., Pereira, M., Rubio, L., \& Weisntein, F. (2015). Pesquisas e manejo de descartes plásticos em sistemas aquáticos uruguaios: atualização e perspectivas. Revista da Gestão Costeira Integrada, 15(3), 377-393. http://dx.doi.org/10.5894/rgci583

Magalhães, S. E. F., \& Araújo, M. C. B. (2012). Lixo marinho na praia de Tamandaré (PE-Brasil):caracterização, análise das fontes e percepção dos usuários da praia sobre o problema. Tropical Oceanography, 40(2), 193208. http://dx.doi.org/10.5914/tropocean.v40i2.5339

Manrich, S., Frattini, G., \& Rosalini, A. C. (1997). Identificação de plásticos: uma ferramenta para reciclagem. São Carlos: UFSCar.

Masura, J., Baker, J., Foster, G., \& Arthur, C. (2015). Laboratory methods for the analysis of microplastics in the marine environment: recommendations for quantifying synthetic particles in waters and sediments (NOAA Technical Memorandum NOS-OR\&R-48). Silver Spring. Recuperado em 26 de janeiro de 2021, de https://marinedebris.noaa.gov/sites/default/files/publicationsfiles/noaa_microplastics_methods_manual.pdf

Montenegro, M., Vianna, M., \& Teles, B. D. (Orgs.). (2020). Atlas do plástico: fatos e números sobre o mundo dos polímeros sintéticos (1. ed.). Rio de Janeiro: Fundação Heirich Böll.

Olivatto, G. P. (2016). Estudo sobre microplásticos em águas superficiais na porção oeste da Baía de Guanabara (Dissertação de mestrado). Curso de Pós-graduação em Química, PUC-Rio.

Pereira, A. M. L., \& Rocha, F. M. R. (2015). A pesca no estuário do Timonha e Ubatuba (PI/CE) (98 p.). Parnaíba: Sieart. 
PlasticsEurope (2016) Plastics - The Facts 2016. Brussels, Belgium: https://www.plasticseurope.org/application/files/4315/1310/4805/plastic-the-fact-2016.pdf

Reisser, J., Shaw, J., Hallegraeff, G., Proietti, M., Barnes, D. K. A., Thums, M., Wilcox, C., Hardesty, B. D., \& Pattiaratchi, C. (2014). Millimeter-sized marine plastics: a new pelagic habitat for microorganisms and invertebrates. PLoS One, 9(6), e100289. http://dx.doi.org/10.1371/journal.pone.0100289

Santos, F. L. B., Torres, M. C., Alves, S. J. S., \& Bezerra, M. M. C. (2020). Avaliação quali-quantitativa de microplásticos em sedimentos e na coluna D’água no Balneário Canto das Águas - Glória/BA e Balneário da Prainha - Paulo Afonso/BA. Brazilian Journal of Development, 6(2), 8439-8453. http://dx.doi.org/10.34117/bjdv6n2-233

Silva, P. H. S., \& Sousa, F. D. B. (2021). Microplastic pollution of Patos Lagoon, south of Brazil. Environmental Challenges, 4, 100076. http://dx.doi.org/10.1016/j.envc.2021.100076

Spinacé, M. A. S., \& Paoli, M. A. (2005). A tecnologia de reciclagem de polímeros. Quimica Nova, 28(1), 65-72. http://dx.doi.org/10.1590/S0100-40422005000100014

Tabajara, L. L. C. A. (1994). Aspectos hidrodinâmicos e sedimentologia do sistema lagunar-estuarino de Tramandaí-RS (Dissertação de mestrado). Universidade Federal do Rio Grande do Sul, Porto Alegre.

Thompson, R. C., Olsen, Y., Mitchelli, R. P., Davis, A., Rowland, S. J., John, A. W. G., Mcgonigle, D., \& Russell, A. E. (2004). Lost at sea: where is all the plastic? Science, 304(5672), 838. http://dx.doi.org/10.1126/science.1094559

Vilela, C. G., Figueira, B. O., Macedo, M. C., \& Baptista Neto, J. A. (2014). Late Holocene evolution and increasing pollution in Guanabara Bay, Rio de Janeiro, SE Brazil. Marine Pollution Bulletin, 79(1-2), 175-187. http://dx.doi.org/10.1016/j.marpolbul.2013.12.020

Vogelmann, E. S., Alegrini, V. R., Awe, G. O., \& Prevedello, J. (2019). Prospects of microplastics in the sedimentary deposits on Patos Lagoon coast. In S. Babel, A. Haarstrick, M. S. Babel \& A. Sharp (Eds.), Water perspectives in emerging countries. Göttingen: Cuvillier Verlag.

Waldschläger, K., Lechthaler, S., Stauch, G., \& Schüttrumpf, H. (2020). The way of microplastic through the environment - application of the source-pathway-receptor model. The Science of the Total Environment, 713, 136584. http://dx.doi.org/10.1016/j.scitotenv.2020.136584

Wang, W., Yuan, W., Chen, Y., \& Wang, J. (2018). Microplastics in surface waters of Dongting Lake and Hong Lake, China. The Science of the Total Environment, 633, 539-545. http://dx.doi.org/10.1016/j.scitotenv.2018.03.211

Wardrop, P., Shimeta, J., Nugegoda, D., Morrison, P. D., Miranda, A., Tang, M., \& Clarke, B. O. (2016). Chemical pollutants sorbed to ingested microbeads from personal care products accumulate in fish. Environmental Science \& Technology, 50(7), 4037-4044. http://dx.doi.org/10.1021/acs.est.5b06280

Woodall, I. C., Sanchez-Vidal, A., Canals, M., Paterson, G. L., Coppock, R., Sleight, V., Calafat, A., Rogers, A. D., Narayanaswamy, B. E., \& Thompson, R. C. (2014). The deep sea is a major sink for microplastic debris. Royal Society Open Science, 1(4), 140371. http://dx.doi.org/10.1098/rsos.140317

Wright, S. L., Thompson, R. C., \& Galloway, T. S. (2013). The physical impacts of microplastics on marine organisms: a review. Environmental Pollution, 178, 483-492. http://dx.doi.org/10.1016/j.envpol.2013.02.031

\section{Contribuições dos autores:}

Laura Steffen Corrêa: coleta, análise e interpretação dos resultados.

Thayse Freitas Silveira: coleta, análise e interpretação dos resultados.

Cacinele Mariana da Rocha: orientação, coleta, coordenação das análises e interpretação dos resultados.

Daiana Maffessoni: orientação, coleta e interpretação dos resultados. 